УДК 517.55

\title{
Successive Approximation for the Inhomogeneous Burgers Equation
}

\author{
Azal Mera* \\ University of Babylon \\ Babylon \\ Iraq \\ Vitaly A. Stepanenko ${ }^{\dagger}$ \\ Institute of Mathematics and Computer Science \\ Siberian Federal University \\ Svobodny, 79, Krasnoyarsk, 660041 \\ Russia \\ Nikolai Tarkhanov ${ }^{\ddagger}$ \\ Institute of Mathematics \\ University of Potsdam \\ Karl-Liebknecht-Str. 24/25, 14476, Potsdam \\ Germany
}

Received 06.04.2017, received in revised form 08.03.2018, accepted 09.06.2018

The inhomogeneous Burgers equation is a simple form of the Navier-Stokes equations. From the analytical point of view, the inhomogeneous form is poorly studied, the complete analytical solution depending closely on the form of the nonhomogeneous term.

\section{Introduction and preliminaries}

The inhomogeneous Burgers equation is the simplest nonlinear model equation for diffusive waves in fluid dynamics. It reads

$$
u_{t}^{\prime}-\nu u_{x x}^{\prime \prime}+u u_{x}^{\prime}=f
$$

where $u$ stands generally for the fluid velocity, $x$ the space variable, $t$ the time variable, $\nu$ is the kinematic viscosity, or the diffusion coefficient, and $f$ a given forcing term. The inverse $R=1 / \nu$ of the diffusion coefficient is known as the Reynold number. Burgers [3] first developed this equation primarily to shed some light on turbulence described by the interaction of two opposite effects of convection and diffusion. However, turbulence is more intricate in the sense that it is both three-dimensional and statistically random in nature. Note that equation (1.1) is parabolic, if $\nu>0$, whereas (1.1) with $\nu=0$ is hyperbolic. More importantly, the properties of solutions to parabolic equations are significantly different from those of hyperbolic equations.

The mathematical structure of equation (1.1) includes a nonlinear convection term $u u_{x}^{\prime}$ which makes the equation more interesting, and a viscosity term of higher order $u_{x x}^{\prime \prime}$ which regularises the equation and produces a dissipation effect of the solution near a shock. When the viscosity

\footnotetext{
*azalmera@gmail.com

†v-stepanen@mail.ru

$\ddagger$ tarkhanov@math.uni-potsdam.de

(c) Siberian Federal University. All rights reserved
} 
coefficient $\nu$ vanishes, the Burgers equation reduces to the transport equation, which represents the inviscid Burgers equation $u_{t}^{\prime}+u u_{x}^{\prime}=f$.

The study of equation (1.1) goes back as far as Forsyth [7] who treated an equation which converts by a change of variables into the Burgers equation. In [1] Bateman introduced the equation (1.1). He was interested in the case where $\nu \rightarrow 0$, and in studying the movement behaviour of a viscous fluid when the viscosity tends to zero. Burgers published a study on equation (1.1) in his paper [3] devoted to turbulence phenomena. Using the transformation discovered later in [6] and independently in [8] Burgers continued his study of state aspects of what he called "nonlinear diffusion equation." The results of this work can be found in [4]. The objective of Burgers was to consider a simplified version of the incompressible NavierStokes equations by neglecting the pressure term. Among the most interesting applications of the Burgers equation in dimension one we mention traffic flow, growth of interfaces, and the financial mathematics, see for instance $[11,18]$.

The nonlinear Burgers equation with $f=0$ can be converted to the linear heat equation and then explicitly solved by the Hopf-Cole transformation. We look for explicit solutions to the forced Burgers equation (1.1), where $f(x, t)$ is the forcing term in a cylinder $\mathcal{C}:=I \times(0, T)$ over a finite interval $I=(a, b)$ of the real axis. In this work we focus on existence, uniqueness and regularity results for the inhomogeneous equation.

For $f=-\lambda w_{x}^{\prime}$, equation (1.1)becomes $u_{t}^{\prime}-\nu u_{x x}^{\prime \prime}+u u_{x}^{\prime}=-\lambda w_{x}^{\prime}$, which is known as the Burgers stochastic equation, where $w=w(x, t)$ stands for the white noise. Using the transformation $u=-\lambda v_{x}^{\prime}$ one sees readily that this is equivalent to the equation

$$
v_{t}^{\prime}-\nu v_{x x}^{\prime \prime}-\frac{\lambda}{2}\left(v_{x}^{\prime}\right)^{2}=w,
$$

which was introduced in [9] and quickly became the default model for random interface growth in physics.

In [2], the main result is the existence and uniqueness of a solution to the inhomogeneous Burgers equation in the anisotropic Sobolev space $H^{2,1}(\mathcal{C})$. This latter is defined to consist of all functions $u \in L^{2}(\mathcal{C})$ whose weak derivatives $\partial_{x}^{\alpha} \partial_{t}^{j} u$ belong to $L^{2}(\mathcal{C})$ for all nonnegative integers $\alpha$ and $j$ satisfying $\alpha+2 j \leqslant 2$. In our paper we develop another approach which has the advantage of being consructive and extends to more general nonlinear problems. It goes back at least as far as [15].

\section{Linearisation}

The change of the unknown function by

$$
u=-2 \nu \frac{U_{x}^{\prime}}{U}=-\nu \frac{\partial}{\partial x} \log U^{2}
$$

reduces the inhomogeneous Burgers equation to

$$
u_{t}^{\prime}-\nu u_{x x}^{\prime \prime}+u u_{x}^{\prime}=-2 \nu \frac{\partial}{\partial x}\left(\frac{U_{t}^{\prime}-\nu U_{x x}^{\prime \prime}}{U}\right)=f
$$

in $\mathcal{C}$. While being intermediate in the Hopf-Cole transformation, the latter equation is equivalent to

$$
U_{t}^{\prime}-\nu U_{x x}^{\prime \prime}+V(x, t) U=0
$$

where $V(x, t)=\frac{1}{2 \nu} \int f(x, t) d x-c(t)$. 
The most familiar boundary value problem for solutions of parabolic equation (1.1) is the first mixed problem

$$
\begin{aligned}
& u(x, 0)=u_{0}(x), \quad \text { if } \quad x \in \bar{I}, \\
& u(x, t)=u_{l}(x, t), \quad \text { if } \quad(x, t) \in \partial I \times[0, T]
\end{aligned}
$$

where we require $u_{0}(x)=u_{l}(x, 0)$ for $x \in \partial I$. The inverse of transformation (2.1) is given by

$$
U(x, t)=C(t) \exp \left(-\frac{1}{2 \nu} \int u(x, t) d x\right),
$$

where $C(t)$ is an arbitrary function of $t$ independent of $x$. Problem (2.3) transforms immediately to the third mixed problem for solutions to parabolic linear equation $(2.2)$

$$
\begin{aligned}
U(x, 0) & =C(0) \exp \left(-\frac{1}{2 \nu} \int u_{0}(x) d x\right), & & \text { if } x \in \bar{I}, \\
U_{x}^{\prime}(x, t)+\frac{1}{2 \nu} u_{l}(x, t) U(x, t) & =0, & & \text { if }(x, t) \in \partial I \times[0, T],
\end{aligned}
$$

the condition on the lateral boundary being of Robin type.

While the transformed Burgers equation given by $(2.2)$ is linear, the coefficient $V(x, t)$ multiplying $U$ is in general a nonsmooth function of $(x, t) \in \mathcal{C}$. Let alone that the boundary conditions become harder to handle. Note that equation (2.2) is of independent interest. In [10], Kac uses the equation to evaluate the Paley-Wiener integral, in which application the coefficient $V(x, t)$ depends solely on $x$. To this end he reduces (2.2) to a Fredholm equation of the second kind which is solved by the Laplace transform. The solution of the integral equation is built in the form of a series expansion over eigenfunction of the equation. In order to establish the convergence of the series Kac first requires the boundedness of $V$ from above, but then he gets rid of this restriction.

In our work we go to treat the inhomogeneous Burgers equation (1.1) directly by reducing it to a nonlinear Fredholm equation of the second kind. This approach can actually be specified within the general framework of nonlinear Fredholm operators in Banach spaces. It leads to existence, uniqueness and regularity theorems provided that the nonlinear term is dominated by the principal linear part of the problem.

\section{Reduction to an integral equation}

Denote by $\psi(x, t)$ the standard fundamental solution of convolution type to the heat equation $u_{t}^{\prime}=\nu u_{x x}^{\prime \prime}$ in $\mathbb{R} \times \mathbb{R}$, i.e.,

$$
\psi(x, t)=\left\{\begin{aligned}
\frac{1}{\sqrt{4 \pi \nu t}} \exp \left(-\frac{x^{2}}{4 \nu t}\right), & \text { if } t>0 \\
0, & \text { if } t \leqslant 0
\end{aligned}\right.
$$

Using the properties of convolution we get $\left(\partial_{t}-\nu \partial_{x}^{2}\right)(\psi * u)=\delta * u=u$ for all distributions $u$ of compact support in $\mathbb{R} \times \mathbb{R}$. As is known, the operator $\Psi u:=\psi * u$ maps distributions of compact support in $\mathbb{R} \times \mathbb{R}$ continuously into distributions in $\mathbb{R} \times \mathbb{R}$.

Rewrite equation (1.1) in the form $u_{t}^{\prime}-\nu u_{x x}^{\prime \prime}=f-N(u)$ in the cylinder $\mathcal{C}$, where $N(u):=u u_{x}^{\prime}$. Assume that $u \in H^{2,1}(\mathcal{C})$ and $f \in L^{2}(\mathcal{C})$. By Theorem 12 of [16] it follows that $u \in C(\overline{\mathcal{C}})$, and so $N(u) \in L^{2}(\mathcal{C})$. Hence, both sides of the equation belong to $L^{2}(\mathcal{C})$. On multiplying both sides of the equation by the characteristic function $\chi_{\mathcal{C}}$ of $\mathcal{C}$ we obtain an equality of distributions of compact support in $\mathbb{R} \times \mathbb{R}$, namely

$$
\chi_{\mathcal{C}}\left(u_{t}^{\prime}-\nu u_{x x}^{\prime \prime}\right)=\left(\partial_{t}-\nu \partial_{x}^{2}\right)\left(\chi_{\mathcal{C}} u\right)+\left[\chi_{\mathcal{C}}, \partial_{t}-\nu \partial_{x}^{2}\right] u=\chi_{\mathcal{C}} f-\chi_{\mathcal{C}} N(u),
$$


where the bracket $\left[\chi_{\mathcal{C}}, \partial_{t}-\nu \partial_{x}^{2}\right]$ stands for the commutator of the operator of multiplication by $\chi_{\mathcal{C}}$ and the heat operator. Applying the fundamental solution $\Psi$ yields

$$
\chi_{\mathcal{C}} u+\Psi\left(\chi_{\mathcal{C}} N(u)\right)=\Psi\left(\chi_{\mathcal{C}} f\right)-\Psi\left(\left[\chi_{\mathcal{C}}, \partial_{t}-\nu \partial_{x}^{2}\right] u\right)
$$

on all of $\mathbb{R} \times \mathbb{R}$. An easy manipulation of the Stokes formula shows that the distribution $\left[\chi_{\mathcal{C}}, \partial_{t}-\right.$ $\left.\nu \partial_{x}^{2}\right] u$ is supported on the boundary of $\mathcal{C}$. To wit,

$$
\begin{aligned}
\left\langle\left[\chi_{\mathcal{C}}, \partial_{t}-\nu \partial_{x}^{2}\right] u, g\right\rangle & =-\int_{\partial \mathcal{C}} u g d x+\nu \int_{\partial \mathcal{C}}\left(u \partial_{x} g-\partial_{x} u g\right) d t= \\
& =\left.\int_{a}^{b} u g\right|_{t=0} ^{t=T} d x+\left.\nu \int_{0}^{T}\left(u \partial_{x} g-\partial_{x} u g\right)\right|_{x=a} ^{x=b} d t
\end{aligned}
$$

holds for all smooth functions $g$ with compact support in $\mathbb{R} \times \mathbb{R}$. We have thus proved the following lemma.

Lemma 3.1. For any function $u \in H^{2,1}(\mathcal{C})$ satisfying the inhomogeneous Burgers equation, we get

$$
u-\mathcal{P}_{s}\left(\partial_{x^{\prime}} u\right)+\mathcal{P}_{v}(N(u))=\mathcal{P}_{i}\left(u_{0}\right)+\mathcal{P}_{d}\left(u_{l}\right)+\mathcal{P}_{v}(f)
$$

where

$$
\begin{array}{ll}
\mathcal{P}_{i}\left(u_{0}\right)=\int_{I} \psi(x-\cdot, t) u_{0} d x^{\prime}, & \mathcal{P}_{s}(v)=\left.\nu \int_{0}^{t} \psi(x-\cdot, t-\cdot) v\right|_{x^{\prime}=a} ^{x^{\prime}=b} d t^{\prime}, \\
\mathcal{P}_{d}\left(u_{l}\right)=-\left.\nu \int_{0}^{t} \partial_{x^{\prime}} \psi(x-\cdot, t-\cdot) u_{l}\right|_{x^{\prime}=a} ^{x^{\prime}=b} d t^{\prime}, & \mathcal{P}_{v}(f)=\int_{I} \int_{0}^{t} \psi(x-\cdot, t-\cdot) f d x^{\prime} d t^{\prime} .
\end{array}
$$

If $I=(-\infty, \infty)$, then both $\mathcal{P}_{s}\left(\partial_{x^{\prime}} u\right)$ and $\mathcal{P}_{d}\left(u_{l}\right)$ vanish and the initial value problem for the inhomogeneous Burgers equation reduces to the nonlinear integral equation of Volterra type

$$
u(x, t)+\int_{I} \int_{0}^{t} \psi(x-\cdot, t-\cdot) N(u) d x^{\prime} d t^{\prime}=\mathcal{P}_{i}\left(u_{0}\right)+\mathcal{P}_{v}(f)
$$

for $(x, t) \in I \times(0, T)$. This equation can be solved by successive approximation, see for instance [10]. In the case of finite intervals $I$ one has to substitute for $\psi$ the Green function of the first mixed problem for the heat equation in the half-strip $I \times(0, \infty)$.

\section{The main theorem}

For $1 \leqslant p \leqslant \infty$ and an integer $s \geqslant 0$, we denote by $L^{p}(I)$ and $H^{s}(I)$ the usual spaces of Lebesgue and Sobolev, respectively. If $\mathcal{B}$ is a Banach space, we write $L^{p}((0, T), \mathcal{B})$ for the space of all measurable functions $u:(0, T) \rightarrow \mathcal{B}$ with the property that

$$
\|u\|_{L^{p}((0, T), \mathcal{B})}=\left(\int_{0}^{T}\|u\|_{\mathcal{B}}^{p} d t\right)^{1 / p}<\infty .
$$

As usual, for $p=\infty$ one substitutes the essential supremum of $\|u\|_{\mathcal{B}}$ on $(0, T)$ for the integral on the right-hand side.

We study the first mixed problem for a class of semilinear parabolic equations which includes, in particular, the Burgers equation in the cylinder $\mathcal{C}$. More precisely, consider

$$
\begin{array}{rlrl}
u_{t}^{\prime}-\nu(t) u_{x x}^{\prime \prime}+\varepsilon(t) u u_{x}^{\prime}+c(x, t) u_{x}^{\prime} & =f & & \text { in } \mathcal{C}, \\
u(x, 0) & =u_{0}(x), & \text { if } x \in I, \\
u(x, t) & =0, & & \text { if } \quad(x, t) \in \partial I \times(0, T),
\end{array}
$$


where $f \in L^{2}(\mathcal{C})$ and $u_{0} \in H_{0}^{1}(I)$ are given functions. The coefficients $\nu(t)$ and $\varepsilon(t)$ are assumed to take on their values in bounded intervals of $\mathbb{R}_{>0}$ away from zero. The coefficient $c(x, t)$ is required to be bounded in some sense in all of $\mathcal{C}$. More precisely, we assume that both $|c(x, t)|$ and $\left|c_{x}^{\prime}(x, t)\right|$ are bounded by a constant $R>0$ for all $(x, t) \in \mathcal{C}$.

Theorem 4.1. Suppose that $f \in L^{2}(\mathcal{C}), u_{0} \in H_{0}^{1}(I)$, and the coefficients $\nu, \varepsilon$ and $c$ satisfy the above conditions. Then problem (4.1) admits a unique solution $u \in H^{2,1}(\mathcal{C})$.

The proof of Theorem 4.1 is based on the Galerkin method. As usual we introduce an approximate solution by reduction to finite-dimensional spaces. By the Galerkin method we establish the existence of an approximation solution, using an existence theorem for a system of ordinary differential equations. We approximate the equation of problem (4.1) by a simpler equation. On using a compactness argument we then make a passage to the limit, thus obtaining the desired solution to (4.1).

\section{Galerkin method}

On multiplying the equation $u_{t}^{\prime}-\nu u_{x x}^{\prime \prime}+\varepsilon u u_{x}^{\prime}+c u_{x}^{\prime}=f$ by a test function $g \in H_{0}^{1}(I)$ and integrating by parts from $a$ to $b$ we get

$$
\int_{a}^{b} u_{t}^{\prime} g d x+\nu(t) \int_{a}^{b} u_{x}^{\prime} g_{x}^{\prime} d x+\varepsilon(t) \int_{a}^{b} u u_{x}^{\prime} g d x+\int_{a}^{b} c(x, t) u_{x}^{\prime} g d x=\int_{a}^{b} f g d x
$$

for almost all $t \in(0, T)$. This is a weak formulation of the differential equation of (4.1). Any function $u \in H^{1}(\mathcal{C})$ satisfying (5.1) and the conditions of (4.1) is called a weak solution to (4.1).

To prove the existence of a weak solution of problem (4.1), we choose an orthonormal basis $\left(e_{j}\right)_{j=1,2, \ldots}$ in $L^{2}(I)$ consisting of the eigenfunctions of $-\partial_{x}^{2}$ for the Dirichlet problem

$$
\begin{aligned}
-\partial_{x}^{2} e_{j} & =\lambda_{j} e_{j} & \text { in } & I, \\
e_{j} & =0 & \text { on } & \partial I
\end{aligned}
$$

in $I$. An easy calculation shows that

$$
\lambda_{j}=\left(\frac{j \pi}{b-a}\right)^{2}, \quad e_{j}(x)=\sqrt{\frac{2}{b-a}} \sin \left(j \pi \frac{x-a}{b-a}\right)
$$

for $j=1,2, \ldots$ Each function $u \in L^{2}(\mathcal{C})$ can be decomposed into the Fourier series

$$
u(x, t)=\sum_{k=1}^{\infty} c_{k}(t) e_{k}(x)
$$

where $c_{k}=\left(u, e_{k}\right)_{L^{2}(I)}$ and the series converges in the $L^{2}(I)$-norm for almost all $t \in(0, T)$.

Given any $n=1,2, \ldots$, we look for an approximate solution $u_{n}$ to problem (4.1) of the form

$$
u_{n}(x, t)=\sum_{k=1}^{n} c_{k}(t) e_{k}(x)
$$

for $(x, t) \in \mathcal{C}$. The coefficients $c_{k}(t)$ depend on $n$, however, we do not display this explicitly by abuse of notation. Since the system $\left(e_{k}\right)_{k=1,2 \ldots}$ is an orthonormal basis of $L^{2}(I)$, it follows that this system is an orthogonal basis of $H_{0}^{1}(I)$. More precisely, the norm of $e_{k}$ in $H^{1}(I)$ just amounts 
to $\sqrt{1+\lambda_{k}}$ and one easily checks that $\left(u, e_{k}\right)_{H^{1}(I)}=\left(1+\lambda_{k}\right)\left(u, e_{k}\right)_{L^{2}(I)}$ for all $k=1,2, \ldots$ By the above, we assume $u_{0} \in H_{0}^{1}(I)$, and so

$$
u_{0}(x)=\sum_{k=1}^{\infty} c_{0, k} e_{k}(x)
$$

on $I$, where $c_{0, k}=\left(u_{0}, e_{k}\right)_{L^{2}(I)}$ and the series converges in the $H^{1}(I)$-norm for almost all $t \in(0, T)$. We require each approximate solution $u_{n}$ to satisfy the system

$$
\begin{aligned}
\int_{a}^{b} \partial_{t} u_{n} e_{j} d x+\nu(t) \int_{a}^{b} \partial_{x} u_{n} \partial_{x} e_{j} d x+\varepsilon(t) \int_{a}^{b} u_{n} \partial_{x} u_{n} e_{j} d x+\int_{a}^{b} c(x, t) \partial_{x} u_{n} e_{j} d x= \\
=\int_{a}^{b} f e_{j} d x \\
u_{n}(\cdot, 0)=\sum_{k=1}^{n} c_{0, k} e_{k}
\end{aligned}
$$

for all $j=1, \ldots, n$ and almost all $t \in(0, T)$.

Remark 5.1. By the very construction, the sequence of initial data $u_{n}(\cdot, 0)$ converges to $u_{0}$ in the Sobolev space $H_{0}^{1}(I)$.

The simple part of Galerkin method consists in establishing that system (5.3) possesses a solution.

Lemma 5.2. For each $n=1,2, \ldots$, system (5.3) admits a unique solution $u_{n}$ of the form (5.2).

Proof. Since $e_{1}, \ldots, e_{n}$ is an orthonormal system in $L^{2}(I)$, we get

$$
\int_{a}^{b} \partial_{t} u_{n} e_{j} d x=\sum_{k=1}^{n} c_{k}^{\prime}(t) \int_{a}^{b} e_{k} e_{j} d x=c_{j}^{\prime}(t)
$$

for all $j=1, \ldots, n$. On the other hand, from the equality $-\partial_{x}^{2} e_{k}=\lambda_{k} e_{k}$ it follows that

$$
\nu(t) \int_{a}^{b} \partial_{x} u_{n} \partial_{x} e_{j} d x=\nu(t) \sum_{k=1}^{n} c_{k}(t) \lambda_{k} \int_{a}^{b} e_{k} e_{j} d x=\nu(t) c_{j}(t) \lambda_{j}
$$

Summing up we see that system (5.3) is equivalent to an initial value problem for the coefficients $c_{1}(t), \ldots, c_{n}(t)$. To wit,

$$
\begin{aligned}
c_{j}^{\prime}+\lambda_{j} \nu(t) c_{j} & =f_{j}(t)-\sum_{k=1}^{n} a_{j, k}(t) c_{k}-\sum_{\substack{k=1, \ldots, n \\
l=1, \ldots, n}} a_{j, k, l}(t) c_{k} c_{l} \text { in }(0, T), \\
c_{j}(0) & =c_{0, j}
\end{aligned}
$$

for $j=1, \ldots, n$, where

$$
\begin{aligned}
a_{j, k}(t) & =\int_{a}^{b} c(x, t) \partial_{x} e_{k} e_{j} d x, \quad a_{j, k, l}(t)=\varepsilon(t) \int_{a}^{b} e_{k} \partial_{x} e_{l} e_{j} d x, \\
f_{j}(t) & =\int_{a}^{b} f e_{j} d x .
\end{aligned}
$$

The left-hand sides of equations (5.4) constitute a system of $n$ uncoupled linear ordinary differential equations. The right-hand sides are well-defined quadratic functions of $c_{1}(t), \ldots, c_{n}(t)$ whose coefficients are integrable functions of $t \in(0, T)$, for $f \in L^{2}(\mathcal{C})$ and $e_{j}, c(x, t)$ are regular. A familiar argument shows that the initial value problem (5.4) has a unique maximal solution defined on some interval $\left[0, T_{n}\right]$ with $T_{n} \leqslant T$. If $T_{n}<T$, then $\left\|u_{n}(\cdot, t)\right\|_{H^{1}(I)}$ must tend to $+\infty$ as $t \rightarrow T_{n}$. The a priori estimates we shall establish later show that this does not happen, and therefore $T_{n}=T$, cf. [17, p. 192]. 


\section{A priori estimate}

In the sequel we use the letters $C_{1}, C_{2}$, etc. to designate diverse constants. They need not be the same in numerious applications unless otherwise stated. As mentioned, we assume

$$
\begin{aligned}
& \nu_{1} \leqslant \nu(t) \leqslant \nu_{2}, \\
& \varepsilon_{1} \leqslant \varepsilon(t) \leqslant \varepsilon_{2}
\end{aligned}
$$

for all $t \in[0, T]$, where $\nu_{j}$ and $\varepsilon_{j}$ are positive constants.

Lemma 6.1. There is a positive constant $C_{1}$ independent of $n$, such that for all $t \in[0, T]$ we get

$$
\left\|u_{n}(\cdot, t)\right\|_{L^{2}(I)}^{2}+\nu_{1} \int_{0}^{t}\left\|\partial_{x} u_{n}(\cdot, s)\right\|_{L^{2}(I)}^{2} d s \leqslant C_{1} .
$$

Proof. Multiplying (5.3) by $c_{j}(t)$ and summing up for $j=1, \ldots, n$ one obtains

$$
\frac{1}{2} \frac{d}{d t} \int_{a}^{b} u_{n}^{2} d x+\nu(t) \int_{a}^{b}\left(\partial_{x} u_{n}\right)^{2} d x-\frac{1}{2} \int_{a}^{b} \partial_{x} c(x, t) u_{n}^{2} d x=\int_{a}^{b} f u_{n} d x
$$

Indeed, because of the boundary conditions one gets

$$
\varepsilon(t) \int_{a}^{b} u_{n}^{2} \partial_{x} u_{n} d x=\varepsilon(t) \int_{a}^{b} \frac{1}{3} \partial_{x}\left(u_{n}\right)^{3} d x=0
$$

and an integration by parts yields

$$
\int_{a}^{b} c(x, t) u_{n} \partial_{x} u_{n} d x=-\frac{1}{2} \int_{a}^{b} \partial_{x} c(x, t) u_{n}^{2} d x .
$$

Then, on integrating in $t$ over $[0, t]$ and using estimates (6.1) one concludes readily that

$$
\begin{aligned}
& \frac{1}{2}\left\|u_{n}(\cdot, t)\right\|_{L^{2}(I)}^{2}+\nu_{1} \int_{0}^{t}\left\|\partial_{x} u_{n}(\cdot, s)\right\|_{L^{2}(I)}^{2} d s \leqslant \\
& \quad \leqslant \frac{1}{2}\left\|u_{n}(\cdot, 0)\right\|_{L^{2}(I)}^{2}+\frac{R}{2} \int_{0}^{t}\left\|u_{n}(\cdot, s)\right\|_{L^{2}(I)}^{2} d s+\int_{0}^{t}\|f(\cdot, s)\|_{L^{2}(I)}\left\|u_{n}(\cdot, s)\right\|_{L^{2}(I)} d s
\end{aligned}
$$

for all $n=1,2, \ldots$

Using the Poincaré inequality

$$
\left\|u_{n}\right\|_{L^{2}(I)}^{2} \leqslant \frac{(b-a)^{2}}{2}\left\|\partial_{x} u_{n}\right\|_{L^{2}(I)}^{2}
$$

along with the elementary inequality

$$
|r s| \leqslant \frac{\epsilon}{2} r^{2}+\frac{1}{2 \epsilon} s^{2}
$$

for $\epsilon=2 \frac{\nu_{1}}{(b-a)^{2}}$ we get

$$
\begin{aligned}
& \left\|u_{n}(\cdot, t)\right\|_{L^{2}(I)}^{2}+\nu_{1} \int_{0}^{t}\left\|\partial_{x} u_{n}(\cdot, s)\right\|_{L^{2}(I)}^{2} d s \leqslant \\
& \quad \leqslant\left\|u_{n}(\cdot, 0)\right\|_{L^{2}(I)}^{2}+R \int_{0}^{t}\left\|u_{n}(\cdot, s)\right\|_{L^{2}(I)}^{2} d s+\frac{(b-a)^{2}}{2 \nu_{1}} \int_{0}^{t}\|f(\cdot, s)\|_{L^{2}(I)}^{2} d s .
\end{aligned}
$$


The second term on the right-hand side of this inequality is obviously dominated by

$$
R \int_{0}^{t}\left(\left\|u_{n}(\cdot, s)\right\|_{L^{2}(I)}^{2}+\nu_{1} \int_{0}^{s}\left\|\partial_{x} u_{n}\left(\cdot, s^{\prime}\right)\right\|_{L^{2}(I)}^{2} d s^{\prime}\right) d s
$$

for all $t \in[0, T]$. Since the sequence $\left(u_{n}(\cdot, 0)\right)_{n=1,2, \ldots}$ converges in $H_{0}^{1}(I)$ to $u_{0}$ (cf. Remark 5.1) and $f \in L^{2}(\mathcal{C})$, there is a positive constant $C$ independent of $n$, such that

$$
\left\|u_{n}(\cdot, 0)\right\|_{L^{2}(I)}^{2}+\frac{(b-a)^{2}}{2 \nu_{1}}\|f\|_{L^{2}(\mathcal{C})}^{2} \leqslant C
$$

whence

$$
\begin{aligned}
& \left\|u_{n}(\cdot, t)\right\|_{L^{2}(I)}^{2}+\nu_{1} \int_{0}^{t}\left\|\partial_{x} u_{n}(\cdot, s)\right\|_{L^{2}(I)}^{2} d s \leqslant \\
& \quad \leqslant C+R \int_{0}^{t}\left(\left\|u_{n}(\cdot, s)\right\|_{L^{2}(I)}^{2}+\nu_{1} \int_{0}^{s}\left\|\partial_{x} u_{n}\left(\cdot, s^{\prime}\right)\right\|_{L^{2}(I)}^{2} d s^{\prime}\right) d s .
\end{aligned}
$$

By the Gronwall inequality,

$$
\left\|u_{n}(\cdot, t)\right\|_{L^{2}(I)}^{2}+\nu_{1} \int_{0}^{t}\left\|\partial_{x} u_{n}(\cdot, s)\right\|_{L^{2}(I)}^{2} d s \leqslant C \exp (R t)
$$

holds for all $t \in[0, T]$. On choosing $C_{1}=C \exp (R T)$ we establish the desired estimate.

The Poincaré inequality shows that our next assertion actually strengthens Lemma 6.1.

Lemma 6.2. There is a positive constant $C_{2}$ independent of $n$, such that for all $t \in[0, T]$ we get

$$
\left\|\partial_{x} u_{n}(\cdot, t)\right\|_{L^{2}(I)}^{2}+\nu_{1} \int_{0}^{t}\left\|\partial_{x}^{2} u_{n}(\cdot, s)\right\|_{L^{2}(I)}^{2} d s \leqslant C_{2} .
$$

Proof. The proof of this a priori estimate is much the same as that of Lemma 6.1.

Lemma 6.3. There is a positive constant $C_{3}$ independent of $n$, such that for all $t \in[0, T]$ we get

$$
\left\|\partial_{t} u_{n}\right\|_{L^{2}(\mathcal{C})}^{2} \leqslant C_{2}
$$

Proof. For any $n=1,2, \ldots$, consider the function

$$
\delta_{n}(x, t)=f(x, t)+\nu(t) \partial_{x}^{2} u_{n}(x, t)-\varepsilon(t) u_{n}(x, t) \partial_{x} u_{n}(x, t)-c(x, t) \partial_{x} u_{n}(x, t)
$$

of $(x, t) \in \mathcal{C}$. To establish that $\partial_{t} u_{n}$ is bounded in $L^{2}(\mathcal{C})$ we will first show that the sequence $\left(\delta_{n}\right)_{n=1,2, \ldots}$ is bounded in $L^{2}(\mathcal{C})$. By the very assumption, we get $f \in L^{2}(\mathcal{C})$. According to Lemmata 6.1 and 6.2 , the terms $c \partial_{x} u_{n}$ and $\nu \partial_{x}^{2} u_{n}$ are bounded in $L^{2}(\mathcal{C})$ uniformly in $n$. It remains only to check that $\varepsilon u_{n} \partial_{x} u_{n}$ is bounded in $L^{2}(\mathcal{C})$ uniformly in $n$.

Lemma 6.1 shows that the norm $\left\|u_{n}\right\|_{L^{\infty}\left([0, T], H_{0}^{1}(I)\right)}^{2}$ is bounded uniformly in $n$. Then, using the embedding of $H_{0}^{1}(I)$ into $L^{\infty}(I)$ yields

$$
\begin{aligned}
& \int_{0}^{T}\left\|\varepsilon u_{n} \partial_{x} u_{n}\right\|_{L^{2}(I)}^{2} d t \leqslant \varepsilon_{2}^{2} \int_{0}^{T}\left(\left\|u_{n}(\cdot, t)\right\|_{L^{\infty}(I)}^{2}\left\|\partial_{x} u_{n}(\cdot, t)\right\|_{L^{2}(I)}^{2}\right) d t \leqslant \\
& \leqslant \varepsilon_{2}^{2} C^{2} \int_{0}^{T}\left(\left\|u_{n}(\cdot, t)\right\|_{H^{1}(I)}^{2}\left\|\partial_{x} u_{n}(\cdot, t)\right\|_{L^{2}(I)}^{2}\right) d t \leqslant \\
& \leqslant\left.\varepsilon_{2}^{2} C^{2}\left\|u_{n}(\cdot, t)\right\|_{L^{\infty}\left([0, T], H^{1}(I)\right)}^{2}\left\|\partial_{x} u_{n}(\cdot, t)\right\|_{L^{2}(\mathcal{C})}^{2}\right) d t \\
&-526-
\end{aligned}
$$


where $C$ is a constant independent of $n$. Hence it follows that $\delta_{n}$ is bounded in $L^{2}(\mathcal{C})$ uniformly in $n$. This already implies that the sequence $\left(\partial_{t} u_{n}\right)_{n=1,2, \ldots}$ is bounded in $L^{2}(\mathcal{C})$.

Indeed, from (5.3) we get

$$
\int_{a}^{b} \partial_{t} u_{n} e_{j} d x=\int_{a}^{b}\left(f+\nu(t) \partial_{x}^{2} u_{n}-\varepsilon(t) u_{n} \partial_{x} u_{n}-c(x, t) \partial_{x} u_{n}\right) e_{j} d x=\int_{a}^{b} \delta_{n} e_{j} d x
$$

for all $j=1, \ldots, n$ and almost all $t \in(0, T)$. On multiplying both sides by $c_{j}^{\prime}(t)$ and summing up for $j=1, \ldots, n$ we obtain

$$
\left\|\partial_{t} u_{n}\right\|_{L^{2}(I)}^{2}=\int_{a}^{b} \delta_{n} \partial_{t} u_{n} d x \leqslant\left\|\delta_{n}\right\|_{L^{2}(I)}\left\|\partial_{t} u_{n}\right\|_{L^{2}(I)}
$$

for all $n=1,2, \ldots$. Hence, $\left\|\partial_{t} u_{n}\right\|_{L^{2}(\mathcal{C})} \leqslant\left\|\delta_{n}\right\|_{L^{2}(\mathcal{C})}$ is bounded uniformly in $n$, as desired.

\section{Existence of a weak solution}

Lemmata 6.1, 6.2 and 6.3 show that the Galerkin approximation $u_{n}$ is bounded in $L^{\infty}\left([0, T], L^{2}(I)\right)$ and $L^{\infty}\left([0, T], H^{2}(I)\right)$, and $\partial_{t} u_{n}$ is bounded in $L^{2}(\mathcal{C})$ uniformly in $n$. So, one can extract a subsequence of $u_{n}$ (we continue to write $u_{n}$ for this subsequence) such that $u_{n} \rightarrow u$ weakly in $L^{2}\left([0, T], H^{2}(I)\right), u_{n} \rightarrow u$ strongly in $L^{2}\left([0, T], L^{2}(I)\right)$ (which just amounts to $L^{2}(\mathcal{C})$ ) and almost everywhere in the rectangle $\mathcal{C}$, and $\partial_{t} u_{n} \rightarrow \partial_{t} u$ strongly in $L^{2}(\mathcal{C})$. Obviously, the limit function $u$ belongs to $H^{2,1}(\mathcal{C})$.

Lemma 7.1. Under the assumptions of Theorem 4.1, problem (4.1) admits a weak solution $u \in H^{2,1}(\mathcal{C})$.

Proof. Since $\partial_{t} u_{n}$ converges to $\partial_{t} u$ in the $L^{2}(\mathcal{C})$-norm, it follows that

$$
\int_{0}^{T} \int_{a}^{b} \partial_{t} u_{n} g d x d t \rightarrow \int_{0}^{T} \int_{a}^{b} \partial_{t} u g d x d t
$$

for all $g \in L^{2}(\mathcal{C})$. On the other hand, as $u_{n}$ converges to $u$ both weakly in $L^{2}\left([0, T], H^{2}(I)\right)$ and in the norm of $L^{2}(\mathcal{C})$, we see that $u_{n} \partial_{x} u_{n}$ converges to $u \partial_{x} u$ weakly in $L^{2}(\mathcal{C})$, and so

$$
\begin{aligned}
& \int_{0}^{T} \int_{a}^{b} \varepsilon(t) u_{n} \partial_{x} u_{n} g d x d t \rightarrow \int_{0}^{T} \int_{a}^{b} \varepsilon(t) u \partial_{x} u g d x d t \\
& \int_{0}^{T} \int_{a}^{b} c(x, t) \partial_{x} u_{n} g d x d t \rightarrow \int_{0}^{T} \int_{a}^{b} c(x, t) \partial_{x} u g d x d t
\end{aligned}
$$

for all $g \in L^{2}(\mathcal{C})$. We make use of these properties when passing to the limit in problem (5.3), as $n \rightarrow \infty$. Given any fixed index $j$, we apply the Fubini theorem to deduce that

$$
\int_{a}^{b} \partial_{t} u e_{j} d x+\nu(t) \int_{a}^{b} \partial_{x} u \partial_{x} e_{j} d x+\varepsilon(t) \int_{a}^{b} u \partial_{x} u e_{j} d x+\int_{a}^{b} c(x, t) \partial_{x} u e_{j} d x=\int_{a}^{b} f e_{j} d x
$$

for almost all $t \in(0, T)$.

Let $g$ be an arbitrary function of $H_{0}^{1}(I)$. Since $\left(e_{j}\right)_{j=1,2, \ldots}$ is an orthogonal basis in $H_{0}^{1}(I)$, the function $g$ can be written as

$$
g=\sum_{j=1}^{\infty} g_{j} e_{j}
$$


where $g_{j}$ are the Fourier coefficients of $g$ with respect to the basis and the series converges in the $H^{1}(I)$-norm. On multiplying the equalities of (7.1) by $g_{j}$, summing up for $j=1, \ldots, N$ and letting $N \rightarrow \infty$ we get (5.1) fulfilled for all $g \in H_{0}^{1}(I)$. By the very construction, $u_{n}(\cdot, 0)$ converges to $u_{0}$ in the $H^{1}(I)$-norm. On the other hand, $u_{n}(x, 0)$ converges to $u(x, 0)$ for almost all $x \in I$, for $u_{n} \rightarrow u$ almost everywhere in $\mathcal{C}$ and $\partial_{t} u_{n} \rightarrow \partial_{t} u$ in the $L^{2}(\mathcal{C})$-norm. It follows that the initial data $u(\cdot, 0)$ of $u$ coincide with $u_{0}$. Moreover, since each function $u_{n}$ belongs to $L^{2}\left([0, T], H_{0}^{1}(I)\right)$ and $u_{n} \rightarrow u$ weakly in $L^{2}\left([0, T], H^{2}(I)\right)$, the limit function $u$ vanishes on the lateral boundary of $\mathcal{C}$. Thus, $u$ is a weak solution of problem (4.1), as desired.

\section{Uniqueness}

Lemma 8.1. Under the assumptions of Theorem 4.1, the solution of problem (4.1) in $H^{2,1}(\mathcal{C})$ is unique.

Proof. We first observe that any solution $u \in H^{2,1}(\mathcal{C})$ of problem $(4.1)$ is in $L^{\infty}\left([0, T], L^{2}(I)\right)$. Indeed, it is easily seen that such a solution $u$ satisfies

$$
\frac{1}{2} \frac{d}{d t} \int_{a}^{b} u^{2} d x+\nu(t) \int_{a}^{b}\left(\partial_{x} u\right)^{2} d x-\frac{1}{2} \int_{a}^{b} \partial_{x} c(x, t) u^{2} d x=\int_{a}^{b} f u d x
$$

for almost all $t \in[0, T]$, because $\int_{a}^{b} u^{2} \partial_{x} u d x=\int_{a}^{b} \frac{1}{3} \partial_{x} u^{3} d x=0$ and

$$
\int_{a}^{b} c(x, t)\left(\partial_{x} u\right) u d x=\int_{a}^{b} c(x, t) \frac{1}{2} \partial_{x} u^{2} d x=-\frac{1}{2} \int_{a}^{b} \partial_{x} c(x, t) u^{2} d x .
$$

Arguing as in the proof of Lemma 6.1 we get

$$
\begin{aligned}
& \|u(\cdot, t)\|_{L^{2}(I)}^{2}+\nu_{1} \int_{0}^{t}\left\|\partial_{x} u(\cdot, s)\right\|_{L^{2}(I)}^{2} d s \leqslant \\
& \quad \leqslant\left\|u_{0}\right\|_{L^{2}(I)}^{2}+R \int_{0}^{t}\|u(\cdot, s)\|_{L^{2}(I)}^{2} d s+\frac{(b-a)^{2}}{2 \nu_{1}} \int_{0}^{t}\|f(\cdot, s)\|_{L^{2}(I)}^{2} d s,
\end{aligned}
$$

and so there is a constant $C>0$ such that

$$
\begin{aligned}
& \|u(\cdot, t)\|_{L^{2}(I)}^{2}+\nu_{1} \int_{0}^{t}\left\|\partial_{x} u(\cdot, s)\right\|_{L^{2}(I)}^{2} d s \leqslant \\
& \quad \leqslant C+R \int_{0}^{t}\left(\|u(\cdot, s)\|_{L^{2}(I)}^{2}+\nu_{1} \int_{0}^{s}\left\|\partial_{x} u\left(\cdot, s^{\prime}\right)\right\|_{L^{2}(I)}^{2} d s^{\prime}\right) d s .
\end{aligned}
$$

By the Gronwall lemma,

$$
\|u(\cdot, t)\|_{L^{2}(I)}^{2}+\nu_{1} \int_{0}^{t}\left\|\partial_{x} u(\cdot, s)\right\|_{L^{2}(I)}^{2} d s \leqslant C \exp (R t)
$$

holds for all $t \in[0, T]$. This shows that $u \in L^{\infty}\left([0, T], L^{2}(I)\right)$ whenever $f \in L^{2}(\mathcal{C})$ and $u_{0} \in L^{2}(I)$.

We now assume that $u_{1}, u_{2} \in H^{2,1}(\mathcal{C})$ are two solutions of problem (4.1). Put $u=u_{1}-u_{2}$. It is clear that $u \in L^{\infty}\left([0, T], L^{2}(I)\right)$. The equations satisfied by $u_{1}$ and $u_{2}$ lead to

$$
\int_{a}^{b}\left(\partial_{t} u g+\nu(t) \partial_{x} u \partial_{x} g+\varepsilon(t) u \partial_{x} u_{1} g+\varepsilon(t) u_{2} \partial_{x} u g+c(x, t) \partial_{x} u g\right) d x=0
$$


for all $g \in H_{0}^{1}(I)$. On choosing $g=u(\cdot, t)$ as a test function, for any fixed $t \in[0, T]$, we deduce that

$$
\begin{aligned}
& \frac{1}{2} \frac{d}{d t}\|u\|_{L^{2}(I)}^{2}+\nu(t)\left\|\partial_{x} u\right\|_{L^{2}(I)}^{2}= \\
& \quad=-\varepsilon(t) \int_{a}^{b} u^{2} \partial_{x} u_{1} d x-\varepsilon(t) \int_{a}^{b} u_{2} u \partial_{x} u d x-\int_{a}^{b} c(x, t) u \partial_{x} u d x
\end{aligned}
$$

An integration by parts yields $\int_{a}^{b} u^{2} \partial_{x} u_{1} d x=-2 \int_{a}^{b} u \partial_{x} u u_{1} d x$, and so (8.1) becomes

$$
\frac{1}{2} \frac{d}{d t}\|u\|_{L^{2}(I)}^{2}+\nu(t)\left\|\partial_{x} u\right\|_{L^{2}(I)}^{2}=\varepsilon(t) \int_{a}^{b}\left(2 u_{1}-u_{2}\right) u \partial_{x} u d x+\frac{1}{2} \int_{a}^{b} \partial_{x} c(x, t) u^{2} d x .
$$

By inequality (6.2) with $\epsilon=2 \nu_{1}$, we get

$$
\begin{aligned}
& \varepsilon(t) \int_{a}^{b}\left(2 u_{1}-u_{2}\right) u \partial_{x} u d x \leqslant \\
& \quad \leqslant \frac{\varepsilon_{2}^{2}}{4 \nu_{1}}\left(2\left\|u_{1}\right\|_{L^{\infty}\left([0, T], L^{2}(I)\right)}+\left\|u_{2}\right\|_{L^{\infty}\left([0, T], L^{2}(I)\right)}\right)^{2}\|u\|_{L^{2}(I)}^{2}+\nu_{1}\left\|\partial_{x} u\right\|_{L^{2}(I)}^{2} .
\end{aligned}
$$

Furthermore,

$$
\left|\int_{a}^{b} \partial_{x} c(x, t) u^{2} d x\right| \leqslant R\|u\|_{L^{2}(I)}^{2}
$$

for all $t \in[0, T]$. Hence it follows that there is a positive constant $C$ with the property that

$$
\frac{1}{2} \frac{d}{d t}\|u\|_{L^{2}(I)}^{2} \leqslant C\|u\|_{L^{2}(I)}^{2}
$$

and so the Gronwall lemma implies $u=0$, as desired.

\section{Boundary value problems in a bounded domain}

The study of boundary value problems for the heat equation was initiated by the familiar paper [13]. In [12], Kondrat'ev developed a general theory of boundary value problems for linear parabolic equations in bounded domains of $\mathbb{R}_{x}^{n} \times \mathbb{R}_{t}$. The focus of [12] is on the asymptotics of solutions at characteristic points of the boundary.

Let $\mathcal{G}$ be the domain in $\mathbb{R}_{x} \times \mathbb{R}_{t}$ consisting of all $(x, t) \in \mathbb{R} \times \mathbb{R}$ with the property that $t \in(0, T)$ and $\varrho_{1}(t)<x<\varrho_{2}(t)$, where $\varrho_{1}$ and $\varrho_{2}$ are functions on $[0, T]$ which are continuously differentiable in the open interval $(0, T)$ and satisfy $\varrho_{1}(t)<\varrho_{2}(t)$ for all $t \in[0, T]$. One looks for a solution to the first mixed problem for the Burgers equation in $\mathcal{G}$, that is

$$
\begin{aligned}
& u_{t}^{\prime}-\nu u_{x x}^{\prime \prime}+u u_{x}^{\prime}=f \quad \text { in } \mathcal{G}, \\
& u(x, 0)=u_{0}(x), \quad \text { if } \quad x \in\left(\varrho_{1}(0), \varrho_{2}(0)\right), \\
& u(x, t)=0, \quad \text { if } \quad(x, t) \in \partial\left(\varrho_{1}(t), \varrho_{2}(t)\right) \times(0, T),
\end{aligned}
$$

where $f \in L^{2}(\mathcal{C})$ and $u_{0} \in H_{0}^{1}\left(\varrho_{1}(0), \varrho_{2}(0)\right)$ are given functions.

Using the results obtained in the foregoing sections, we look for conditions on the functions $\varrho_{1}$ and $\varrho_{2}$ which guarantee that problem $(9.1)$ admits a unique solution $u \in H^{2,1}(\mathcal{G})$. In order to solve problem (9.1), we apply the method which was used, e.g., in [14] and [5]. This method consists in establishing that the problem admits a unique solution when the domain $\mathcal{G}$ is transformed into a rectangle by means of a suitable change of variables preserving the anisotropic Sobolev space $H^{2,1}(\mathcal{G})$. 
Theorem 9.1. If $f \in L^{2}(\mathcal{C}), u_{0} \in H_{0}^{1}\left(\varrho_{1}(0), \varrho_{2}(0)\right)$ and $\left|\varrho_{2}^{\prime}(t)-\varrho_{1}^{\prime}(t)\right| \leqslant C$ for all $t \in(0, T)$, then problem (9.1) has precisely one solution in $H^{2,1}(\mathcal{G})$.

The proof of this assertion invokes an appropriate change of variables which allows one to use Theorem 4.1.

Proof. Namely, consider the mapping $h: \mathcal{G} \rightarrow \mathbb{R} \times R$ given by

$$
y=\frac{x-\varphi_{1}(t)}{\varphi_{2}(t)-\varphi_{1}(t)}, \quad s=t
$$

for $(x, t) \in \mathcal{G}$. This mapping transforms $\mathcal{G}$ into the rectangle $\mathcal{C}=(0,1) \times(0, T)$. Setting $u(x, t)=v(y, s)$ and $f(x, t)=g(y, s)$, we rewrite (9.1) equivalently in the form

$$
\begin{aligned}
v_{s}^{\prime}-\frac{\nu}{(\varphi(s))^{2}} v_{y y}^{\prime \prime}+\frac{1}{\varphi(s)} v v_{y}^{\prime}-\frac{\varphi^{\prime}(s) y+\varphi_{1}^{\prime}(s)}{\varphi(s)} v_{y}^{\prime} & =g \quad \text { in } \quad \mathcal{C}, \\
v(\cdot, 0) & =v_{0} \quad \text { on } I, \\
v & =0 \quad \text { on } \quad \partial I \times(0, T),
\end{aligned}
$$

where $I=(0,1), \varphi=\varphi_{2}-\varphi_{1}$ and $v_{0}(y)=u_{0}\left(\varphi(0) y+\varphi_{1}(0)\right)$. It is easily verified that the change of variables $(y, s)=h(x, t)$ preserves the spaces $H_{0}^{1}, H^{2,1}$ and $L^{2}$. Moreover, the conditions on the coefficients of (9.2) imposed in Section 4. are fulfilled. Therefore, we are now in the setting of problem (4.1), and so the desired result follows from Theorem 4.1.

Theorem 9.1 can be generalised to the case where $\varphi_{1}$ and $\varphi_{2}$ are Lipschitz continuous functions on $[0, T]$ instead of $C^{1}(0, T)$. On the other hand, the question arises what happens if $\varphi_{1}(0)=\varphi_{2}(0)$. In this latter case $\left(\varphi_{1}(0), 0\right)$ is an isolated singular point at the boundary of $\mathcal{G}$. The study of problem (9.1) near this point would require analysis in domains with conical or more generally cuspidal points at the boundary.

The first author gratefully acknowledges the financial support of the Ministry of High Education of Iraq.

\section{References}

[1] H.Bateman, Some recent researches on the motion of fluid, Mon. Weather Rev., 43(1915), $163-170$.

[2] Y.Benia, B.-K.Sadallah, Existence of solutions to Burgers equation in domains that can be transformed into rectangles, Electr. J. Diff. Eq., 157(2016), 1-13.

[3] J.M.Burgers, A mathematical model illustrating the theory of turbulence, Adv. Appl. Mech., 1(1948), 171-199.

[4] J.M.Burgers, The Nonlinear Diffusion Equation, Reidel, Dordrecht, 1974.

[5] H.R.Clark, M.A.Rincon, A.Silva, Analysis and numerical simulation of viscous Burgers equation, Numer. Funct. Anal. and Optim., 32(2011), no. 7, 695-716.

[6] J.D.Cole, On a quasilinear parabolic equation occurring in aerodynamics, Quart. Appl. Math., 9(1951), 225-236.

[7] A.R.Forsyth, Theory of Differential Equations, Part 4: Partial Differential Equations, Cambridge University Press, Cambridge, 1906. 
[8] E.Hopf, The partial differential equation $u_{t}+u_{x} u=\mu u_{x x}$, Comm. Pure Appl. Math. 3(1950), 201-230.

[9] M.Kardar, G.Parisi, Y.-C.Zhang, On a quasilinear parabolic equation occurring in aerodynamics, Phys. Rev. Lett., 56 (1986), 889-892.

[10] M.Kac, Probability and Related Topics in the Physical Sciences, Interscience Publishers, UK, 1959.

[11] J.Kevorkian, Partial Differential Equations: Analytical Solution Techniques, Brooks/Cole Publ. Pacific Grove, California, 1990.

[12] V.A.Kondrat'ev, Boundary problems for parabolic equations in closed domains, Trans. Moscow Math. Soc., 15(1966), 450-504.

[13] I.G.Petrovskii, On the solution of the first boundary value problem for the heat equation, Uch. Zapiski MGU, 2(1934), 55-59 (in Russian).

[14] B.-K.Sadallah, Étude d'un problème $2 m$-parabolique dans des domaines plan non rectangulaires, Boll. U.M.I., 6(1983), 51-112.

[15] A.Shlapunov, N.Tarkhanov, An open mapping theorem for the Navier-Stokes equations, Prepr. Inst. of Math. Univ. of Potsdam, 5(2016), no. 10, 1-80.

[16] L.N.Slobodetskij, The generalised spaces of S.L. Sobolev and their application to boundary value problems for partial differential equations, Uch. Zap. Leningr. Ped. Inst. im. A. I. Gertsena, 197(1958), 54-112 (in Russian).

[17] R.Temam, Navier-Stokes Equations, AMS, Providence, RI, 1984.

[18] G.W.Whitham, Lectures on Wave Propagation, Narosa Publ. House, New Dehli, 1979.

\section{Последовательное приближение для неоднородного уравнения Бюргерса}

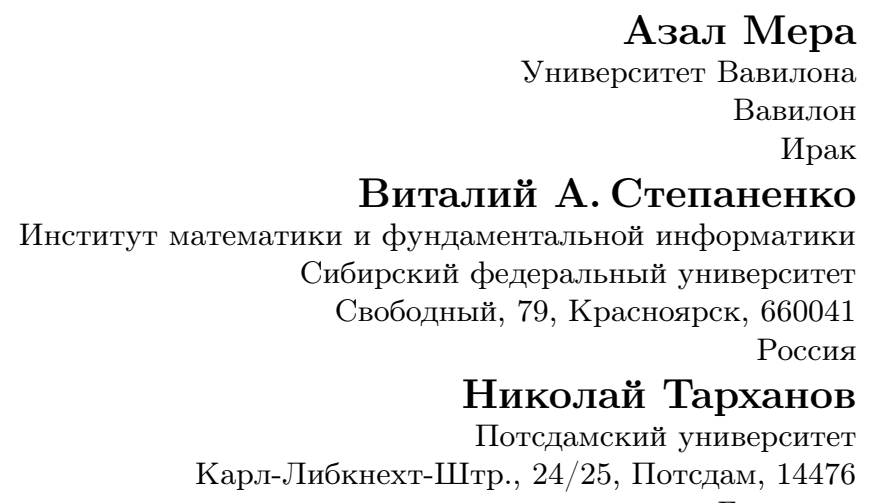

Германия

Неоднородное уравнение Бюргерса представляет собой простой вид уравнений Навъе-Стокса. $C$ аналитической точки зрения неоднородная форма плохо изучена, а полное аналитическое решение тесно зависит от формы неоднородного члена.

Ключевые слова: уравнения Навъе-Стокса, классическое решение. 